\title{
Longitudinal Mode Competition in Chirped Grating Distributed Feedback Lasers
}

\author{
Kim A. Winick, Senior Member, IEEE
}

\begin{abstract}
Gain competition often inhibits the simultaneous lasing of multiple longitudinal modes in homogeneously broadened laser systems. A stability analysis is developed to demonstrate that the two lowest order degenerate longitudinal modes in an indexcoupled distributed feedback (DFB) laser will lase simultaneously when the index grating is chirped asymmetrically along the axis of the device. This chirped grating structure is shown to decrease gain competition by reducing the spatial overlap between the degenerate modes. Stable mode beating between the two lowest order lasing modes results, and this beating produces high-frequency output self-pulsations which can be used for millimeter-wave and soliton pulse train generation. An exact closed-form expression for the output intensity of an antisymmetrically chirped index-coupled DFB laser, as a function of the unsaturated gain, is also derived. The expression is valid for arbitrary levels of gain saturation.
\end{abstract}

Index Terms - Chirped grating, distributed feedback (DFB) laser, mode competition, waveguide laser.

\section{INTRODUCTION}

$\mathbf{I}$ T IS WELL known that the two lowest order modes of a uniform index-coupled distributed feedback (DFB) laser are degenerate and symmetrically located about the grating stopband [1]. Experimental results indicate that single-mode operation of these devices is possible, though multimode behavior is often observed. Zhang et al. explained these observations by numerically solving the time-dependent DFB laser coupled-mode equations in the presence of envelope gain saturation and population-dependent refractive index variations [2]. Using a similar approach, Liao and Winful [3] and Wake [4] recently predicted, theoretically, that an antisymmetrically chirped single-section DFB laser can undergo sustained high-frequency self-pulsations of the output beam intensity. These are not true monomode pulsations [5] but rather the beating between two degenerate modes supported simultaneously by the chirped-grating DFB structure. Fessent also observed, numerically, that multimode behavior can occur in some corrugation-pitch-modulated DFB lasers [6]. Dual-frequency optical sources have a number of potentially important applications, including millimeter-wave [7]-[9] and soliton pulse train generation [10]. It is the purpose of this paper to demonstrate, analytically, that an antisymmetricallychirped DFB laser can operate stably, above threshold, in two degenerate modes.

Manuscript received March 16, 1999; revised June 28, 1999. This work was supported in part by the National Science Foundation under Grant ECS9522200 .

The author is with the Electrical Engineering and Computer Science Department, University of Michigan, Ann Arbor, MI 48109 USA.

Publisher Item Identifier S 0018-9197(99)07610-1.
DFB lasers have been studied extensively since the first such devices were experimentally demonstrated in 1971 [11], [12]. In their classic paper, Kogelnik and Shank analyzed the threshold behavior of these devices using steady-state coupledmode theory [1]. Their analysis yielded formulas for the lasing thresholds, lasing frequencies, and longitudinal mode intensity profiles. Analytic expressions for above-threshold operation, however, are difficult to obtain, because envelope gain saturation results in nonlinear behavior. Hill and Watanabe determined the steady-state performance of DFB lasers operating above threshold by numerically solving the coupledmode equations [13]. Single-frequency operation was assumed and spatial and spectral hole burning were neglected. Haus obtained similar results, but did so using an approximate analytic technique [14]. Szczepanski later modified Haus's basic approach to improve its accuracy [15], [16]. Solimeno and Mastrocinque also developed some analytic results for gain-coupled DFB lasers operating at low levels of envelope gain saturation [17].

Equations describing the above-threshold steady-state operation of DFB lasers in the presence of spatial hole burning, due to cavity standing waves, were derived starting in the mid1970's [18]-[21]. In [21], Rabinovich et al. combined these equations with Haus's analytic approach to get approximate closed-form solutions for the laser's output power versus unsaturated gain. Rabinovich also studied the degree to which spatial hole burning effects the suppression of higher order longitudinal modes. None of these papers, however, addressed the issue of multimode stability in DFB lasers.

The remainder of this paper is organized as follows. In Section II, the properties of the degenerate modes of an antisymmetrically chirped index-coupled DFB laser operating at threshold are described. In Section III, a stability analysis is presented to show that stable dual-mode operation is possible for these lasers. In Section IV, the time-dependent coupled mode equations for chirped-grating index-coupled DFB lasers are solved numerically. The results are shown to support, quantitatively, our previously derived analytic results. Finally, our conclusions are presented in Section V.

\section{Mode Properties IN ASYMMETRICALly CHIRPED, INDEX-COUPLED DFB LASERS}

In this section, we will demonstrate that: 1) the lowest order modes of a linearly chirped index-coupled DFB laser are degenerate and symmetrically located, in frequency, about the grating stopband; 2) the mode intensity profiles of these two modes are mirror images of one another with respect to a 


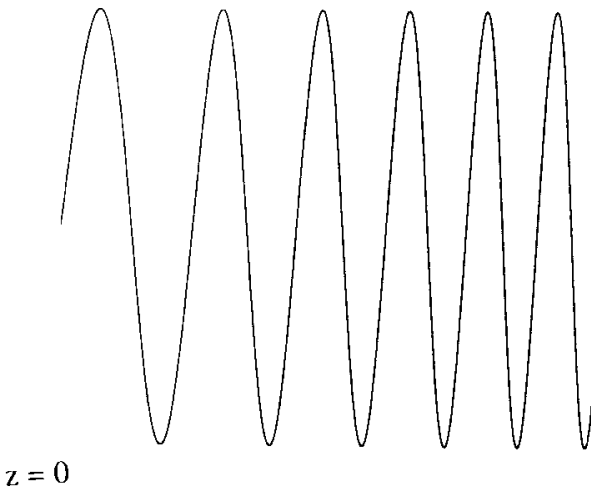

$z=0$

Fig. 1. Grating with antisymmetric linear chirp.

plane located at the center of the cavity; 3) the spatial overlap integral between the two modes is less than the self-overlap integral of either mode individually; and 4) the cavity $Q$ is the same for both modes. We start our analysis by considering an index-coupled DFB laser having a refractive index variation $n(z)$ of the following form:

$$
n(z)=n_{o}+n_{1}(z) \cos \left(\frac{2 \pi}{\Lambda} z+\phi(z)\right)
$$

with $n_{1} \ll n_{o}$. We will assume that the mode profile transverse to the direction of propagation $z$ is uniform with a cross-sectional area of $A$. The time-independent coupled-mode equations can then be written as [1]

$$
\begin{gathered}
\frac{d R(z)}{d z}+(-\alpha(z)+i \delta) R(z)=-i \kappa(z) S(z) \\
\frac{d S(z)}{d z}-(-\alpha(z)+i \delta) S(z)=i \kappa^{*}(z) R(z) \\
\delta=\frac{n_{o} \omega}{c}-\frac{\pi}{\Lambda} \\
\kappa(z)=\frac{\omega}{c} \frac{n_{1}(z)}{2} \exp (-i \phi(z))
\end{gathered}
$$

where $R(z)$ and $S(z)$ are the electric field amplitudes of the forward $(+z)$ and backward $(-z)$ propagating beams of frequency $\omega$, respectively, $\kappa(z)$ is the strength of the spatially varying coupling coefficient, $\phi(z)$ is the phase of the grating chirp, $\Lambda$ is the nominal period of the grating, $\alpha(z)$ is the net saturated amplitude gain, $c$ is the vacuum speed of light, and $\delta$ is the deviation from the nominal Bragg condition. In general, $\alpha$ will be a function of $z$, due to nonuniform pumping and gain saturation. In this section, we will assume that the pumping is uniform and that gain saturation is weak. Therefore, $\alpha$ will be taken to be a constant independent of $z$. The laser is assumed to be of length $L$, with perfectly antireflection-coated end facets located at $z=0$ and $z=L$. Thus, the boundary conditions for lasing become

$$
R(0)=S(L)=0 .
$$

The normalized mode frequency $(\delta L)_{k}$ and the corresponding gain threshold $(\alpha L)_{k}$ of the $k$ th mode can be found numerically using (2.2)-(2.6). We will number the modes according to the convention $0<(\delta L)_{1}<(\delta L)_{2}<(\delta L)_{3} \cdots$ and $0>(\delta L)_{-1}>(\delta L)_{-2}>(\delta L)_{-3}$. If the grating chirp is antisymmetric (see Fig. 1), i.e.,

$$
\phi(L-z)=\phi(z)
$$

or equivalently

$$
\frac{d \phi(L-z)}{d z}=-\frac{d \phi(z)}{d z}
$$

and the laser is operating near threshold (i.e., $\alpha \approx$ net unsaturated gain), then (2.2), (2.3), and (2.7) can be combined to yield

$$
\begin{aligned}
\frac{d S^{*}(L-z)}{d z}+ & (-\alpha-i \delta) S^{*}(L-z) \\
& =-i \kappa(z)\left(-R^{*}(L-z)\right) \\
\frac{d\left(-R^{*}(L-z)\right)}{d z}- & (-\alpha-i \delta)\left(-R^{*}(L-z)\right) \\
= & i \kappa^{*}(z) S^{*}(L-z) .
\end{aligned}
$$

Comparing (2.2) and (2.3) with (2.8) and (2.9) and invoking the boundary conditions (2.6), we see that, if $R_{k}(z)$ and $S_{k}(z)$ correspond to the forward and backward propagating beams of laser mode $k$, then $S_{k}^{*}(L-z)$ and $-R_{k}^{*}(L-z)$ correspond to the forward and backward propagating beams of laser mode $-k$, where $\delta_{k}=\delta_{-k}$. Thus

$$
\begin{aligned}
R_{-k}(z) & =S_{k}^{*}(L-z) \\
S_{-k}(z) & =-R_{k}^{*}(L-z) \\
\delta_{-k} & =\delta_{k} .
\end{aligned}
$$

For purposes of illustration, the normalized lasing mode frequencies $(\delta L)_{k}$ and the corresponding threshold amplitude gain-length product $(\alpha L)_{k}$ were found numerically using (2.2)-(2.6). DFB lasers with unapodized coupling strengths (i.e., $|\kappa L|=$ constant independent of $z$ ) and antisymmetric linear chirps described by

$$
\phi(z)=g_{c}\left(\frac{2 z}{L}-1\right)^{2}, \quad 0 \leq z \leq 1
$$

were assumed. The chirp parameter $g_{c}$ is a constant which determines the amount of chirp present. The results are shown in Fig. 2 for the cases $|\kappa L|=2, g_{c}=0$ (i.e., no chirp), and $|\kappa L|=2, g_{c}=2$, respectively. Note that in both cases, pairs of degenerate modes are located symmetrically with respect to 


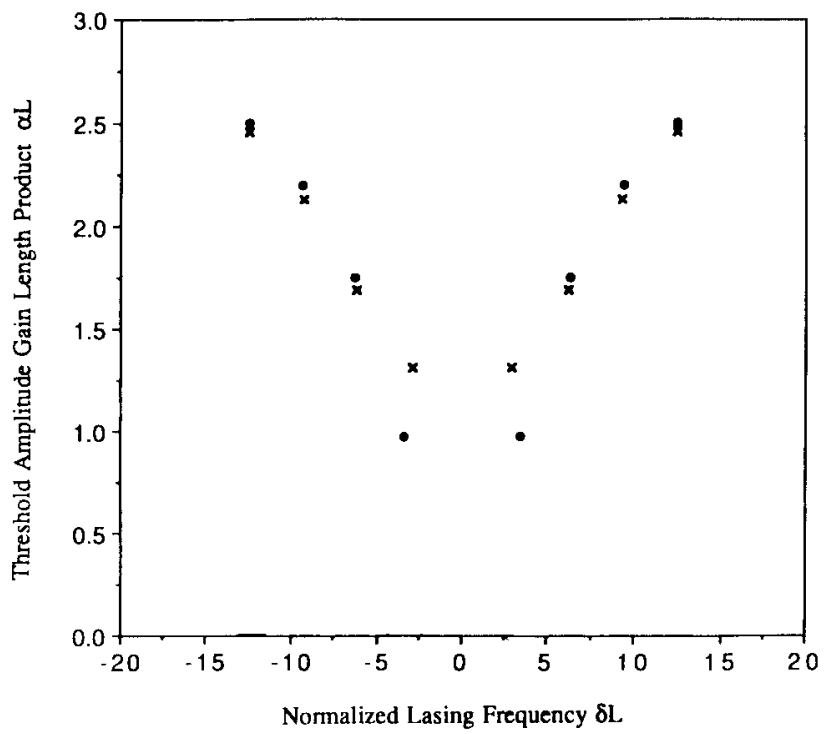

Fig. 2. Lasing frequency and threshold amplitude gain. (a) $x:|\kappa L|=2$, $g_{c}=2$. (b) $\bullet:|\kappa L|=2, g_{c}=0$.

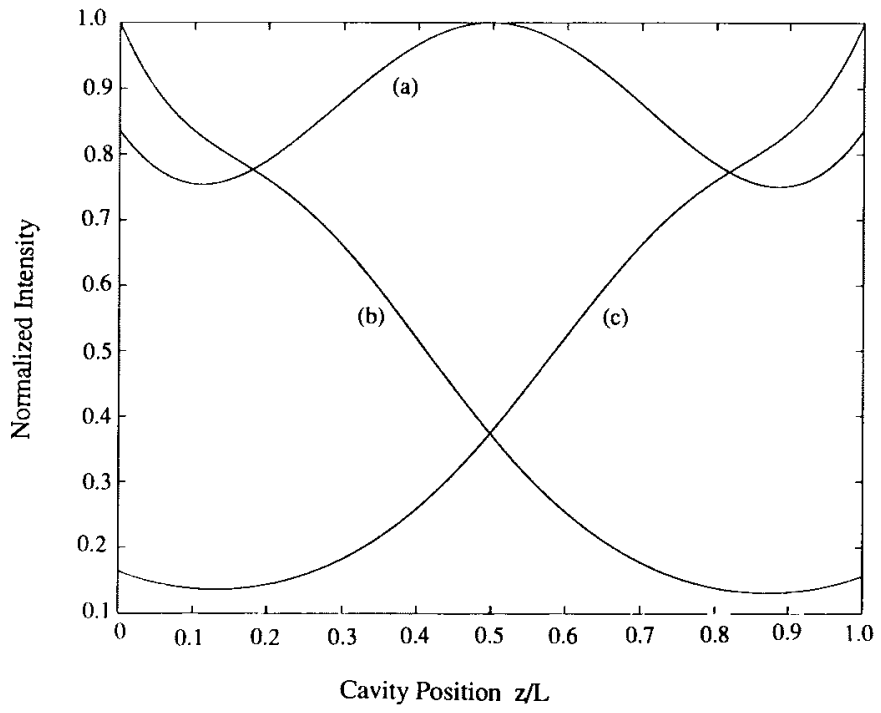

Fig. 3. Normalized mode intensities at threshold. (a) $|\kappa L|=2, g_{c}=0$, $\delta L= \pm 3.37, \alpha L=0.98$. (b) $|\kappa L|=2, g_{c}=2, \delta L=2.92, \alpha L=1.31$ (c) $|\kappa L|=2, g_{c}=2, \delta L=-2.92, \alpha L=1.31$.

the stopband $\delta L=0$. In Fig. 3, the envelope intensity profiles $\left|R_{k}(z)\right|^{2}+\left|S_{k}(z)\right|^{2}$ of the lowest order pair of degenerate modes (i.e., $k= \pm 1$ ) are plotted for the cases shown in Fig. 2.

If the two degenerate +1 and -1 modes lase simultaneously, then the beat frequency $\Delta f=f_{+}-f_{-}$in hertz is found from (2.4) to be

$$
\Delta f=\frac{|\delta L|_{1}}{\pi\left(\frac{n_{o} L}{c}\right)} .
$$

Furthermore, the output power $P_{\text {out }}(t)$ will be proportional to

$$
\begin{aligned}
P_{\text {out }}^{ \pm}(t) \propto & \left|R_{1}(L) \exp \left(i \omega_{1} t\right) \pm R_{-1}(L) \exp \left(i \omega_{-1} t\right)\right|^{2} \\
= & \left|R_{1}(L) \exp \left(i \omega_{1} t\right) \pm S_{1}^{*}(0) \exp \left(i \omega_{-1} t\right)\right|^{2} \\
= & \left|R_{1}(L)\right|^{2}+\left|S_{1}(0)\right|^{2} \pm 2\left|R_{1}(L) S_{1}(0)\right| \\
& \cdot \cos (2 \pi \Delta f t+\theta)
\end{aligned}
$$

TABLE I

Lasing FreQuency, Threshold, AND Modulation Depth versus Chirp $(|\kappa L|=2)$

\begin{tabular}{cccc}
\hline $\begin{array}{c}\text { chirp coefficient } \\
g_{c}\end{array}$ & $(\delta L)_{\text {thresh }}$ & $(\alpha L)_{\text {thresh }}$ & $\begin{array}{c}\text { modulation depth } \\
m_{d}\end{array}$ \\
\hline 0.0 & 3.37 & 0.98 & $100 \%$ \\
0.5 & 3.34 & 1.0 & $96.9 \%$ \\
1.0 & 3.26 & 1.06 & $88.3 \%$ \\
2.0 & 2.92 & 1.31 & $69.6 \%$ \\
3.0 & 2.39 & 1.63 & $52.4 \%$ \\
4.0 & 1.73 & 1.95 & $56.4 \%$ \\
5.0 & 1.09 & 2.25 & $74 \%$ \\
\hline
\end{tabular}

where the plus sign in (2.15) indicates the power emitted from the laser end facet located at $z=L$, while the minus sign corresponds to that emitted from the end facet at $z=0$. Note that the oscillating terms in (2.15) are $180^{\circ}$ out of phase at the two end facets. It also follows from (2.15) that the modulation depth $m_{d}$ of the beat frequency is given by

$$
m_{d}=\frac{2\left|R_{1}(L) S_{1}(0)\right|}{\left|R_{1}(L)\right|^{2}+\left|S_{1}(0)\right|^{2}} .
$$

Equation (2.16) and Fig. 3 indicate that the modulation depth for the linearly chirped case $|\kappa L|=2$ and $g_{c}=2$ is approximately $70 \%$. In general, for fixed $|\kappa L|$, numerical calculations show that the beat frequency decreases while the threshold gain increases as the chirp $g_{c}$ gets larger. Initially, the modulation depth also decreases. This general trend is illustrated in Table I.

We now consider the spatial overlap integral between the two lowest order degenerate modes. Two separate cases will be examined, one with spatial hole burning and the other without. In the absence of spatial hole burning, the cavity beam intensities of the +1 and -1 modes are proportional to $g_{1}(z)$ and $g_{-1}(z)$, respectively, given as

$$
\begin{aligned}
g_{1}(z) & =\left|R_{1}(z)\right|^{2}+\left|S_{1}(z)\right|^{2} \\
g_{-1}(z) & =\left|S_{1}^{*}(L-z)\right|^{2}+\left|-R_{1}^{*}(L-z)\right|^{2} \\
& =g_{1}(L-z) .
\end{aligned}
$$

Thus, the beam intensities of the +1 and -1 degenerate modes are mirror images of one another as seen from the midpoint $z=L / 2$ of the laser cavity.

Note that for any real valued functions $g_{1}(z)$ and $g_{-1}(z)$

$$
\begin{aligned}
0 & \leq \int_{0}^{L}\left(g_{1}(z)-g_{-1}(z)\right)^{2} d z \\
& =\int_{0}^{L} g_{1}^{2}(z) d z+\int_{0}^{L} g_{-1}^{2}(z) d z-2 \int_{0}^{L} g_{1}(z) g_{-1}(z) d z
\end{aligned}
$$

Combining (2.18) and (2.19) now yields

$$
\begin{aligned}
\int_{0}^{L} g_{1}^{2}(z) d z & =\int_{0}^{L} g_{-1}^{2}(z) d z \\
& \geq \int_{0}^{L} g_{1}(z) g_{-1}(z) d z
\end{aligned}
$$

where the second inequality in (2.20) is satisfied with equality if and only if $g_{1}(z)=g_{-1}(z)$. Equation (2.20) implies that 
the spatial overlap between the +1 and -1 modes is less than the self overlap of either of these modes individually. Next we show that (2.20) remains valid in the presence of spatial hole burning. For this case, the +1 and -1 mode intensities are proportional to $g_{1}(z)$ and $g_{-1}(z)$, respectively, given as

$$
\begin{aligned}
g_{1}(z)= & \left|R_{1}(z)\right|^{2}+\left|S_{1}(z)\right|^{2}+2 \operatorname{Re}\left(R_{1}(z) S_{1}^{*}(z)\right) \\
g_{-1}(z)= & \left|S_{1}^{*}(L-z)\right|^{2}+\left|-R_{1}^{*}(L-z)\right|^{2} \\
& -2 \operatorname{Re}\left(R_{1}(L-z) S_{1}^{*}(L-z)\right) .
\end{aligned}
$$

The third terms on the right-hand sides of (2.21) and (2.22) are quasi-periodic with a period much less than $L$. Thus,

$$
\int_{0}^{L}\left(\left|R_{1}(z)\right|^{2}+\left|S_{1}(z)\right|^{2}\right) \operatorname{Re}\left(R_{1}(z) S_{1}^{*}(z)\right) d z \approx 0 .
$$

Combining (2.19) and (2.21)-(2.23) once again yields (2.20). Equation (2.20) will be used in Section III to show that the simultaneous stable lasing of the +1 and -1 degenerate laser modes is possible for any antisymmetrically chirped indexcoupled DFB laser.

The photon decay rate $\gamma$ in a laser cavity is inversely proportional to the $Q$ of the cavity and depends on the cavity losses and the stored cavity energy. In particular,

$$
\begin{aligned}
& \gamma= \\
& \frac{(\text { output power })+(\text { power lost through parasitic effects })}{\text { stored cavity energy }}
\end{aligned}
$$

The three terms appearing on the right-hand side of (2.24) are given by

$$
\begin{aligned}
& \text { output power }=\frac{A}{2 Z}\left[\left|R_{k}(L)\right|^{2}+\left|S_{k}(0)\right|^{2}\right] \\
& \text { parasitic loss }=\frac{A \alpha_{L}}{Z} \int_{0}^{L}\left[\left|R_{k}(z)\right|^{2}+\left|S_{k}(z)\right|^{2}\right] d z \\
& \text { stored energy }=\frac{A \varepsilon}{2} \int_{0}^{L}\left[\left|R_{k}(z)\right|^{2}+\left|S_{k}(z)\right|^{2}\right] d z
\end{aligned}
$$

where $2 \alpha_{L}=$ is the power loss propagation coefficient, $Z$ is the characteristic impedance of the laser material, $\varepsilon$ is the permittivity of the laser material, and $A$ is the cross-sectional area of the mode. We will define the function $f_{k}(z)$ to be a normalized unit-less quantity, which is proportional to the mode intensity in the cavity. Thus,

$$
\begin{gathered}
f_{k}(z) \propto\left|R_{k}(z)\right|^{2}+\left|S_{k}(z)\right|^{2} \\
\int_{0}^{L} f_{k}(z) d z / L=1
\end{gathered}
$$

Combining (2.24)-(2.29) yields

$$
\gamma_{k}=2 \alpha_{L k} v_{p k}+\frac{V_{p k}}{L}\left(f_{k}(L)+f_{k}(0)\right)
$$

where $v_{p}$ is the phase velocity in the laser material. Since the frequency spacing between the +1 and -1 degenerate modes is relatively small, we will assume that

$$
\alpha_{L 1} \approx \alpha_{L(-1)} \text { and } \quad v_{p 1} \approx v_{p(-1)}
$$

Combining (2.10), (2.11), (2.28), (2.30), and (2.31) yields

$$
\gamma_{1} \approx \gamma_{-1}
$$

Note that the results of this section were derived assuming laser operation near threshold and, therefore, small gain saturation. It has been observed elsewhere, however, that the longitudinal mode profiles do not change appreciably from their threshold values as output power is increased provided $|\kappa L|>0.2$ [15]. In semiconductor lasers, the refractive index and, hence, the effective grating period depends on the population inversion. Thus, in the presence of gain saturation, where the population inversion depends on the longitudinal position along the cavity length, the grating phase profile $\phi(z)$ will depend on both the pitch of the index corrugation and the mode intensities. If the chirp of the corrugation pitch is sufficiently large, however, we expect that the mode intensity dependency will be a second-order effect. Finally, we note that spatial hole burning effects are only significant in solid-state lasers, since in semiconductor lasers carrier diffusion tends to smear out rapid spatial variations in gain.

\section{Stability ANALYSIS}

In this section, we will demonstrate that the two lowest order degenerate modes of any antisymmetrically chirped DFB laser can lase simultaneously. Our results will be obtained by modifying the stability analysis first proposed by Lamb to study gain competition in Doppler broadened lasers [22], [23]. We will make a number of simplifying assumptions in our analysis in order to make the problem tractable. We will assume that: 1) the laser is a solid-state device; 2) the mode profile is uniform in a plane transverse to the direction of propagation; 3 ) the emission corresponds to a four-level purely homogeneously broadened transition; 4) the unsaturated population inversion is uniform along the length of the cavity; $5)$ the population inversion is only weakly to moderately saturated; and 6) the beat frequency is sufficiently high that the population inversion is unresponsive to it. In practice, this last assumption is almost always satisfied.

The rate equation for the cavity photon density (photons/unit volume) $p_{k}(z, t)$ corresponding to the $k$ th mode $(k= \pm 1)$ can be written as

$$
\frac{d p_{k}(z, t)}{d t}=\sigma_{k} v_{p k} p_{k}(z, t) N(z, t)-\gamma_{k} p_{k}(z, t)
$$

where $v_{p k}$ is the phase velocity $c / n_{o}, \sigma_{k}$ is the emission cross section, $\gamma_{k}$ is the cavity decay rate, and $N(z, t)$ is the population inversion per unit volume. If $P_{k}(t)$ denotes the total number of cavity photons in mode $k$ and $A$ the cross-section area of the mode, then we can write

$$
p_{k}(z, t)=\frac{P_{k}(t)}{A L} f_{k}(z)
$$

where $f_{k}(z)$ is proportional to the longitudinal dependence of the intensity of the $k$ th mode and is given by (2.28) and (2.29). Since the frequency spacing between the +1 and -1 degenerate modes is relatively small, we will assume that the 
emission cross sections and phase velocities do not depend on $k$. Thus, we will write

$$
\begin{gathered}
\sigma_{1} \approx \sigma_{-1} \approx \sigma \\
v_{p 1} \approx v_{p(-1)}=v_{p} .
\end{gathered}
$$

Integrating both sides of (3.1) with respect to $z$ and using (3.2) yields

$$
\begin{aligned}
\frac{d P_{k}(t)}{d t} & =\int_{0}^{L} \sigma v_{p} p_{k}(z, t) N(z, t) d z A-\gamma P_{k}(t) \\
& =\int_{0}^{L} \sigma v_{p} P_{k}(t) \frac{f_{k}(z)}{L} N(z, t) d z-\gamma P_{k}(t) .
\end{aligned}
$$

For an ideal four-level system, the population inversion $N(z, t)$ is described by the following rate equation:

$$
\begin{aligned}
\frac{\partial N(z, t)}{\partial t}= & R_{p}-\sigma v_{p}\left(p_{1}(z, t)+p_{-1}(z, t)\right) N(z, t) \\
& -\frac{N(z, t)}{\tau_{f}} \\
= & R_{p}-\frac{\sigma v_{p}}{A L}\left(P_{1}(t) f_{1}(z)+P_{-1}(t) f_{-1}(z)\right) N(z, t) \\
& -\frac{N(z, t)}{\tau_{f}}
\end{aligned}
$$

where $R_{p}$ is the pump rate into the upper lasing level and $\tau_{f}$ is the fluorescence lifetime of this level. The number of cavity photons in each mode and the population inversion will be written as

$$
\begin{aligned}
P_{1}(t) & =P_{1}^{s s}+\varepsilon_{1}(t) \\
P_{-1}(t) & =P_{-1}^{s s}+\varepsilon_{-1}(t) \\
N(z, t) & =N^{s s}(z)+\Delta N(z, t)
\end{aligned}
$$

where $\varepsilon_{1}(t), \varepsilon_{-1}(t)$, and $\Delta N(z, t)$ denote deviations from the steady-state values $P_{1}^{s s}, P_{-1}^{s s}$, and $N^{s s}(z)$. Combining (3.6)-(3.9) yields

$$
\begin{aligned}
& \tau_{f} \frac{\partial \Delta N(z, t)}{\partial}+\left(\frac{P_{1}^{s s}}{P_{\text {sat }}} f_{1}(z)+\frac{P_{-1}^{s s}}{P_{\text {sat }}} f_{-1}(z)+1\right) \Delta N(z, t) \\
&+\left(\frac{\varepsilon_{1}(t)}{P_{\text {sat }}} f_{1}(z)+\frac{\varepsilon_{-1}(t)}{P_{\text {sat }}} f_{-1}(z)\right) N^{s s}(z)=0
\end{aligned}
$$

and

$$
N^{s s}(z)=\frac{N_{o}}{1+\frac{P_{1}^{s s} f_{1}(z)+P_{-1}^{s s} f_{-1}(z)}{P_{\text {sat }}}}
$$

where

$$
P_{\mathrm{sat}}=\frac{A L}{\sigma v_{p} \tau_{f}}
$$

and $N_{\circ}$ is the unsaturated population inversion. Taking the Laplace transform of (3.10) yields

$$
\Delta N(z, s)=\frac{-1}{\tau_{f} s+1}\left(f_{1}(z) \frac{\varepsilon_{1}(s)}{P_{\mathrm{sat}}}+f_{-1}(z) \frac{\varepsilon_{-1}(s)}{P_{\mathrm{sat}}}\right) N_{o}
$$

where we have neglected the saturation terms $P_{1}^{s s} / P_{\text {sat }}$ and $P_{-1}^{s s} / P_{\text {sat }}$, which to first order are assumed to be small. Under the same assumption, (3.11) can be written as

$$
N^{s s}(z) \approx N_{o}\left(1-f_{1}(z) \frac{P_{1}^{s s}}{P_{\text {sat }}}-f_{-1}(z) \frac{P_{-1}^{s s}}{P_{\text {sat }}}\right) .
$$

Combining (3.5), (3.7)-(3.9), and (3.14) yields

$$
\begin{aligned}
\frac{d P_{1}(t)}{d t}= & \int_{0}^{L} \frac{N_{o} \sigma v_{p}}{L}\left(P_{1}^{s s}+\varepsilon_{1}(t)\right) f_{1}(z) \\
& \cdot\left[1-\frac{P_{1}^{s s}}{P_{\text {sat }}} f_{1}(z)-\frac{P_{-1}^{s s}}{P_{\text {sat }}} f_{-1}(z)+\frac{\Delta N(z, t)}{N_{o}}\right] d z \\
& -\gamma\left(P_{1}^{s s}+\varepsilon_{1}(t)\right) \\
\frac{d P_{-1}(t)}{d t}= & \int_{0}^{L} \frac{N_{o} \sigma v_{p}}{L}\left(P_{-1}^{s s}+\varepsilon_{-1}(t)\right) f_{-1}(z) \\
& \cdot\left[1-\frac{P_{1}^{s s}}{P_{\text {sat }}} f_{1}(z)-\frac{P_{-1}^{s s}}{P_{\text {sat }}} f_{-1}(z)+\frac{\Delta N(z, t)}{N_{o}}\right] d z \\
& -\gamma\left(P_{-1}^{s s}+\varepsilon_{-1}(t)\right) .
\end{aligned}
$$

At steady state, the right-hand sides of (3.15) and (3.16) are zero as are the terms $\varepsilon_{1}(t), \varepsilon_{-1}(t)$, and $\Delta N(z, t)$. Thus, we obtain the following two simultaneous equations for $P_{1}^{s s}$ and $P_{-1}^{s s}$ :

$$
\begin{aligned}
& \frac{\Gamma a}{P_{\text {sat }}} P_{1}^{s s}+\frac{\Gamma b}{P_{\text {sat }}} P_{-1}^{s s}+(\gamma-\Gamma)=0 \\
& \frac{\Gamma b}{P_{\text {sat }}} P_{1}^{s s}+\frac{\Gamma a}{P_{\text {sat }}} P_{-1}^{s s}+(\gamma-\Gamma)=0
\end{aligned}
$$

where

$$
\begin{aligned}
a & =\int_{0}^{L} f_{1}^{2}(z) d z / L \\
b & =\int_{0}^{L} f_{1}(z) f_{-1}(z) d z / L \\
\Gamma & =N_{\circ} \sigma v_{p} .
\end{aligned}
$$

From (2.20), we conclude that

$$
b<a
$$

for any antisymmetrically chirped index-coupled DFB laser. Note that lasing requires that the unsaturated gain exceeds the cavity losses, and thus

$$
\Gamma=N_{o} \sigma v_{p}>\gamma
$$

It follows from (3.17) and (3.18) that the steady-state number of cavity photons in each of the two degenerate +1 and -1 modes, denoted $\left(P_{1}^{s s}, P_{-1}^{s s}\right)$, can assume only one of three possible pairs of values as shown in Fig. 4:

$$
\begin{aligned}
& P_{1}^{s s}=\frac{P_{\text {sat }}\left(1-\frac{\gamma}{\Gamma}\right)}{a} \text { and } P_{-1}^{s s}=0 \\
& P_{-1}^{s s}=\frac{P_{\text {sat }}\left(1-\frac{\gamma}{\Gamma}\right)}{a} \text { and } P_{1}^{s s}=0 \\
& P_{1}^{s s}=P_{-1}^{s s}=\frac{P_{\text {sat }}\left(1-\frac{\gamma}{\Gamma}\right)}{a+b} .
\end{aligned}
$$




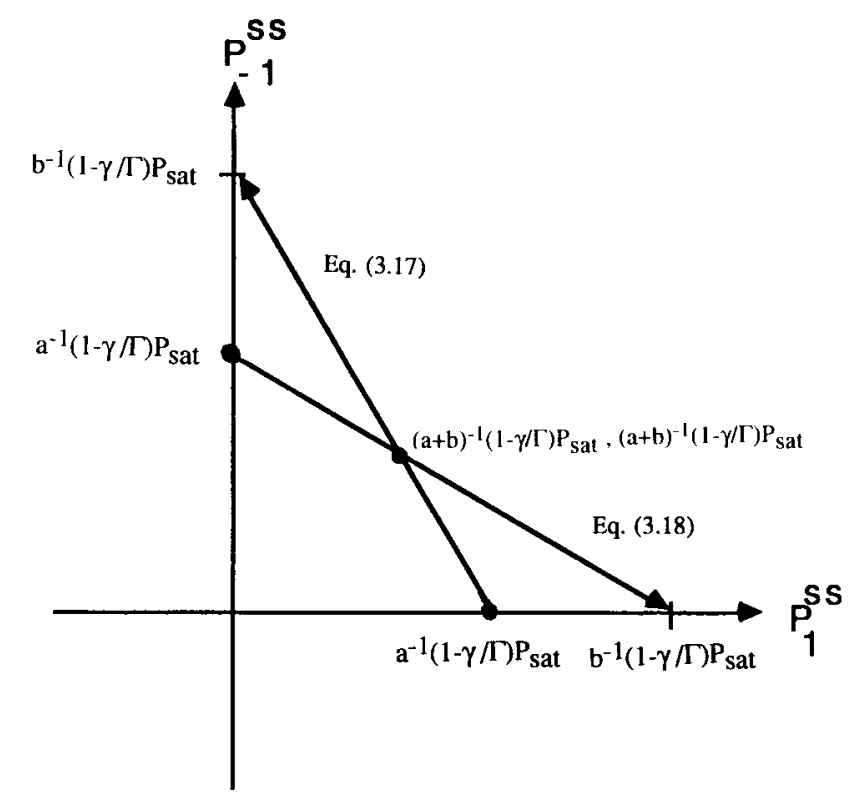

Fig. 4. Steady-state solutions $(\bullet)$.

Equations (3.23) and (3.24) represent single-mode or bistable operation, whereas (3.25) represents dual-mode lasing. We will now determine whether single-mode and/or dual-mode operation is stable under perturbations [23]. We consider first the dual-mode case and substitute (3.7)-(3.9) and (3.25) into (3.15) and (3.16). Taking the Laplace transform of the two resulting equations and using (3.13) yields

$$
\begin{aligned}
\tau_{f} s^{2} \varepsilon_{1}(s)+(s+A) \varepsilon_{1}(s)+B \varepsilon_{-1}(s) & =0 \\
\tau_{f} s^{2} \varepsilon_{-1}(s)+(s+A) \varepsilon_{-1}(s)+B \varepsilon_{1}(s) & =0
\end{aligned}
$$

where

$$
\begin{aligned}
& A=(\Gamma-\gamma) \frac{a}{a+b} \\
& B=(\Gamma-\gamma) \frac{b}{a+b}
\end{aligned}
$$

The roots $s_{1}, s_{2}, s_{3}$, and $s_{4}$ of the characteristic equation

$$
\left(\tau_{f} s^{2}+s+A\right)^{2}-B^{2}=0
$$

associated with (3.26) and (3.27) are given by

$$
s=\frac{-1 \pm \sqrt{1-4(A \pm B) \tau_{f}}}{2 \tau_{f}} .
$$

Thus,

$$
\begin{aligned}
\varepsilon_{1}(t) & =\sum_{m=1}^{4} c_{m} e^{s_{m} t} \\
\varepsilon_{-1}(t) & =\sum_{m=1}^{4} d_{m} e^{s_{m} t}
\end{aligned}
$$

where the $c_{m}$ 's and $d_{m}$ 's are constants. Since $A>B$ for any antisymmetrically chirped grating by (3.21), (3.22), (3.28), and (3.29), the poles $s_{1}, s_{2}, s_{3}$, and $s_{4}$ lie strictly in the left half plane. Thus, $\varepsilon_{1}(t)$ and $\varepsilon_{-1}(t)$ must decay to zero as $t \rightarrow \infty$; therefore, simultaneous stable operation of the +1 and -1 modes is possible. As the value of $a$ approaches $b$, a pair of poles moves closer to the $j \omega$ axis. Thus, the system becomes less stable as the spatial overlap between the +1 and -1 modes increases. Similarly, we can analyze the stability of singlemode operation. Substituting (3.7)-(3.9) and (3.23) into (3.15) and (3.16) and taking the Laplace transform yields

$$
\begin{array}{r}
\tau_{f} s^{2}+(s+\Gamma-\gamma) \varepsilon_{1}(s)+\frac{b}{a}(\Gamma-\gamma) \varepsilon_{-1}(s)=0 \\
s \varepsilon_{-1}(s)+\left(\frac{b}{a}-1\right)(\Gamma-\gamma) \varepsilon_{-1}(s)=0
\end{array}
$$

It is clear that this system will be stable when $\Gamma-\gamma>0$ if and only if $b>a$. Thus, single-mode operation of any antisymmetrically chirped index-coupled DFB laser is not stable. An ideal uniform (i.e., unchirped) index-coupled DFB laser will have $a=b$. Therefore, neither single- nor dualmode operation will be strictly stable for this type of device. In this section, we developed results for any antisymmetrically chirped index-coupled DFB laser. The analysis technique presented, however, is valid for any four-level laser system having a pair of degenerate modes.

We can also use our results to derive an exact expression for the total average output power $P_{\text {out }}$ of the laser as a function of the unsaturated amplitude gain $\alpha_{\text {unsat }}=\sigma N_{o} / 2$. By symmetry,

$$
P_{1}^{s s}=P_{2}^{s s}
$$

Thus,

$$
P_{\text {out }}=2 P_{1}^{s s}\left[f_{1}(0)+f_{1}(L)\right] v_{p} h \nu_{s} / L
$$

where $\nu_{s}$ is the nominal lasing frequency. In steady state, the derivative in (3.5) is zero, and thus this equation reduces to

$$
\frac{\sigma v_{p}}{L} \int_{0}^{L} f_{1}(z) N(z) d z=\gamma
$$

Combining (3.11) and (3.38) yields

$$
\frac{\sigma v_{p} N_{o}}{L} \int_{0}^{L} \frac{f_{1}(z)}{1+\frac{P_{1}^{s s}}{P_{\text {sat }}}\left[f_{1}(z)+f_{1}(L-z)\right]} d z=\gamma
$$

From (3.37) and (3.12), we have

$$
\frac{P_{1}^{s s}}{P_{\text {sat }}}=\frac{P_{\text {out }}}{A \frac{h \nu_{s}}{\sigma \tau_{f}}} \frac{1}{2\left[f_{1}(0)+f_{1}(L)\right]} .
$$

Combining (3.39), (3.40), and (2.30) yields our final result

$$
\begin{gathered}
\left(\frac{1}{2} \sigma N_{o}\right) \int_{0}^{L} \frac{f_{1}(z)}{1+\frac{P_{\text {out }}}{A \frac{h \nu_{s}}{\sigma \tau_{f}}} \frac{f_{1}(z)+f_{1}(L-z)}{2\left[f_{1}(0)+f_{1}(L)\right]}} d z \\
=\alpha_{L} L+\frac{1}{2}\left[f_{1}(0)+f_{1}(L)\right]
\end{gathered}
$$

This expression gives the unsaturated amplitude gain $\alpha_{\text {unsat }}=$ $\sigma N_{o} / 2$ as a function of the output power $P_{\text {out }}$ of the laser. Note that (3.41) is valid for arbitrarily high levels of gain saturation. Evaluation of (3.41) requires knowledge of the mode intensity profile $f_{1}(z)$. This profile, however, is not 
a strong function of the unsaturated gain, and thus it can be approximated by the mode intensity profile at threshold. Equation (3.14) is an extension to two-mode operation of a result first reported by Haus [13].

\section{Time-Dependent Coupled-Mode Equations}

In the previous section, we demonstrated that an antisymmetrically chirped index-coupled DFB laser can support the simultaneous stable lasing of two degenerate modes. We derived an expression for the resulting beat frequency, (2.14), and modulation depth, (2.16), of the beat. In principle, the results of our analysis could be verified by numerical simulations [3], [24]. If population dynamics are to be included in the simulation, however, the computational load becomes very high. This is especially true for solid-state lasers, because upper level lifetimes may be long, and, thus, long time histories must be simulated before steady-state conditions are reached. Spatial hole burning effects can also be included in the simulations, if desired, using the technique described by Rabinovich [21]. In this section, we will neglect spatial hole burning effects due to cavity standing waves and will assume relatively short upper state lifetimes in order to make the simulations less time-consuming. The simulation results will be shown to support our earlier analytic results.

Consider an index-coupled DFB laser with refractive index variation $n(z)$ given by (2.1). We express the total timedependent electric field $E(z, t)$ in the laser as

$$
\begin{aligned}
E(z, t)=E_{F}(z, t) \exp & \left(-i\left(\beta_{o} z-\omega t\right)\right) \\
& +E_{B}(z, t) \exp \left[\left(i\left(\beta_{o} z+\omega t\right)\right)\right]
\end{aligned}
$$

where

$$
\beta_{o}=\frac{\pi}{\Lambda}
$$

In (4.1), $\omega=2 \pi \nu_{s}$ is the nominal laser oscillation frequency, $E_{F}$ is the electric field of the forward $(+z)$ propagating beam in the cavity, and $E_{B}$ is the electric field of the backward $(-z)$ propagating beam. In the absence of spatial hole burning, the time-dependent coupled-mode equations for a chirped-grating index-coupled DFB laser can be written as [25]

$$
\begin{aligned}
& \frac{n_{o}}{c} \frac{\partial E_{F}(z, t)}{\partial t}+\frac{\partial E_{F}(z, t)}{\partial z} \\
& =-i \kappa_{B F} E_{B}(z, t)+(\alpha(z, t)-i \delta) E_{F}(z, t) \\
& \frac{n_{o}}{c} \frac{\partial E_{B}(z, t)}{\partial t}-\frac{\partial E_{B}(z, t)}{\partial z} \\
& =-i \kappa_{F B}(z) E_{F}(z, t)+(\alpha(z, t)-i \delta) E_{B}(z, t)
\end{aligned}
$$

where

$$
\begin{aligned}
\kappa_{B F}(z) & =\frac{\omega}{c} \frac{n_{1}}{2} \exp (-i \phi(z)) \\
\kappa_{F B}(z) & =\kappa_{B F}^{*}(z) \\
\delta & =\frac{n_{o} \omega}{c}-\beta_{o} .
\end{aligned}
$$

The net saturated amplitude gain coefficient $\alpha(z, t)$ is given by

$$
\alpha(z, t)=\frac{\sigma N(z, t)}{2}-\alpha_{L}
$$

The population inversion $N(z, t)$ in the absence of spatial hole burning is described by the following rate equation:

$$
\frac{\partial N(z, t)}{\partial t}=R_{p}-\frac{2 \pi}{h \omega} \sigma \frac{\left|E_{F}(z, t)\right|^{2}+\left|E_{B}(z, t)\right|^{2}}{2 Z}-\frac{N(z, t)}{\tau_{f}}
$$

where $R_{p}$ is the pump rate into the upper lasing level and $\tau_{f}$ is the fluorescence lifetime of this level. The coupledmode equations (4.3), (4.4), and (4.9) can be easily solved numerically using the methods of characteristics [23]. By making the following change of variables:

$$
\begin{gathered}
\eta=\frac{z+\frac{c}{n_{o}} t}{2 L} \\
\tau=\frac{\frac{c}{n_{o}} t-z}{2 L}
\end{gathered}
$$

the mixed derivative coupled-mode equations become the following ordinary differential equations:

$$
\begin{aligned}
\frac{\partial E_{F}(\eta, \tau)}{\partial \eta}= & -i\left(\kappa_{B F}(z) L\right) E_{B}(\eta, \tau) \\
& +(\alpha(N(\eta, \tau)) L-i(\delta L)) E_{F}(\eta, \tau) \\
\frac{\partial E_{B}(\eta, \tau)}{\partial \tau}= & -i\left(\kappa_{F B}(z) L\right) E_{F}(\eta, \tau) \\
& +(\alpha(N(\eta, \tau)) L-i(\delta L)) E_{B}(\eta, \tau)
\end{aligned}
$$

Note that the net saturated amplitude gain $\alpha$ is a function of the population inversion $N$, and this dependence is included in (4.12) and (4.13) by the term $\alpha(N)$. We have solved the three coupled equations (4.9), (4.12), and (4.13) numerically for a linearly chirped antisymmetric grating described by (2.13). A uniform coupling coefficient, $\left|\kappa_{F B} L\right|=2$, was assumed along with three different values of chirp $g_{c}=0$ (i.e., no chirp), $g_{c}=1$, and $g_{c}=2$. Parasitic losses were set to zero, i.e., $\alpha_{L}=0$. The time it takes to make one transit of the cavity $t_{c}$ is given by

$$
t_{c}=\frac{n_{o} L}{c}
$$

Thus, it follows from Table I and (2.14) that the beat frequency is approximately equal to $1 / t_{c}$ for $\left|\kappa_{F B} L\right|=2$. Our analysis in this paper is predicated on the assumption that changes of the population inversion occur too slowly to follow the rapid time variations of the cavity intensity caused by mode beating. Thus, in our simulations, we must choose $\tau_{f} / t_{c}>1$ in order to satisfy this constraint. Larger values of $\tau_{f} / t_{c}$, however, require longer simulation runs before steady-state conditions are reached. Thus, as a compromise, we have chosen the value of $\tau_{f} / t_{c}$ to be 1 . This choice allows the saturation of the population inversion to vary rapidly, but not fast enough to follow the beat frequency. Finally, a constant complex number, whose real and imaginary parts are Gaussian and independently distributed with mean zero and variance $10^{-4}$, is added to the right-hand sides of both (4.12) and (4.13) for the first ten or so cavity transits time. This noise process is 


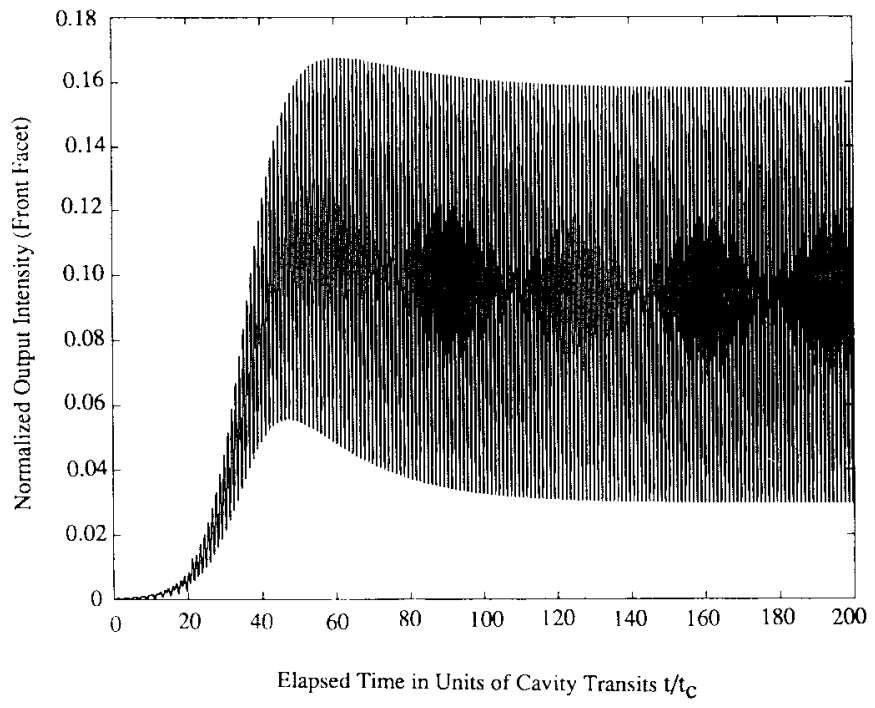

Fig. 5. Normalized output beam intensity from front facet $(|\kappa L|=2$, $\left.g_{c}=2,(\alpha L)_{\text {unsat }}=1.4\right)$.

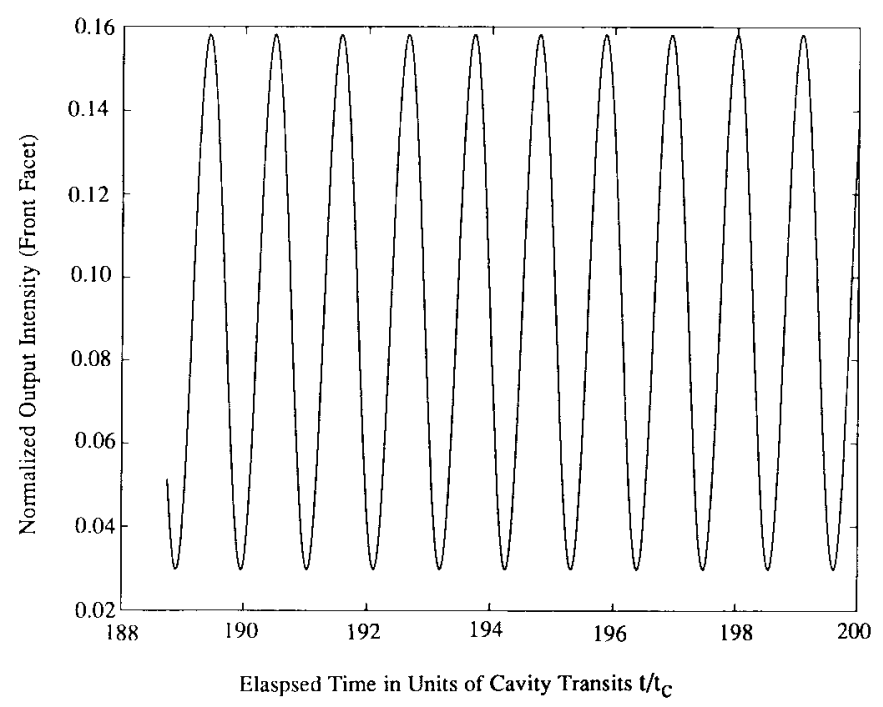

Fig. 6. Normalized output beam intensity from front facet $(|\kappa L|=2$, $\left.g_{c}=2,(\alpha L)_{\text {unsat }}=1.4\right)$.

used to "seed" the fields and allow lasing action to build up, much as spontaneous emission noise does in an actual laser.

In Figs. 5 and 6 , the output beam intensity at the $z=$ 0 end facet is plotted as a function of time, measured in units of cavity transits. The plotted intensity is normalized to the saturation intensity, $h \nu_{s} /\left(\sigma \tau_{f}\right)$. An unsaturated amplitude gain-length product $\alpha_{\text {unsat }} L$ of 1.4 was assumed together with $\left|\kappa_{F B} L\right|=2$ and a chirp factor $g_{c}=2$. Note that the unsaturated gain-length product is only slightly above threshold, as indicated in Table I. We observe that after approximately 100 cavity transits stable oscillations are observed with a modulation depth of approximately $68 \%$. A spectral analysis of the field amplitude indicates the presence of two frequency components located at $\delta L= \pm 2.92$ in agreement with theory (see Table I). The observed modulation depth is also in close agreement with theory as Table I indicates. Fig. 7 shows the time evolution of the saturated amplitude gain-length product

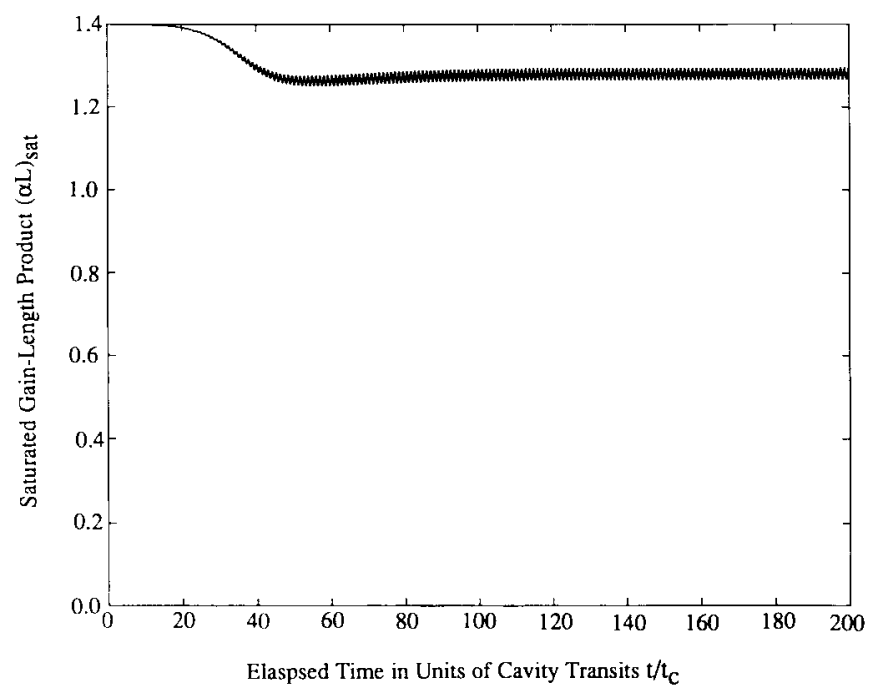

Fig. 7. Saturated gain-length product at front facet $\left(|\kappa L|=2, g_{c}=2\right.$, $\left.(\alpha L)_{\text {unsat }}=1.4\right)$.

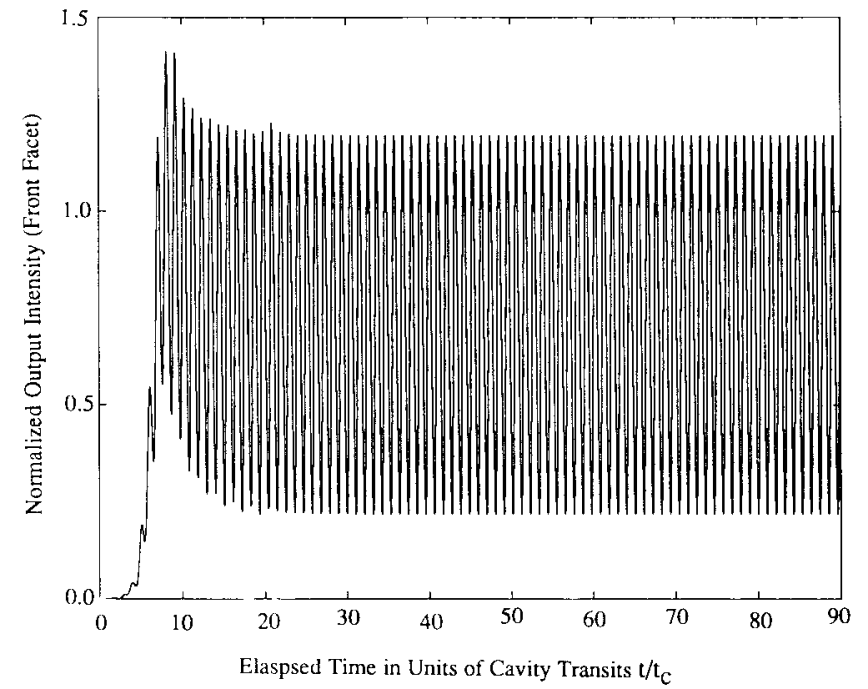

Fig. 8. Normalized output beam intensity from front facet $(|\kappa L|=2$, $\left.g_{c}=2,(\alpha L)_{\text {unsat }}=2.0\right)$.

$\alpha_{\mathrm{sat}} L$ at the $z=0$ end facet. Note the presence of a slight ringing, which we have observed disappears when the value of $\tau_{f} / t_{c}$ is increased. Simulation results are shown in Fig. 8 under identical conditions, but with an unsaturated gain-length product that is considerably above threshold. Note that similar results are obtained for this case, even though our analysis, which was developed for the weakly to moderately saturated case, does not strictly apply. Fig. 9 shows simulation results for a grating with a very weak chirp, i.e., $g_{c}=0.5$. Mode beating commences after approximately 20 cavity transit times. The modulation depth is initially small and increases slowly. In steady state, our theory predicts that a modulation depth of close to 1 will result. The slow increase in the modulation depth is consistent with our theory, since for this case the mode overlap between the +1 and -1 modes is large. This gives rise to poles in our stability analysis which lie close to the $j \omega$ axis and, hence, indicates a slow response time. Finally, Fig. 10 show simulation results for an ideal unchirped grating, 


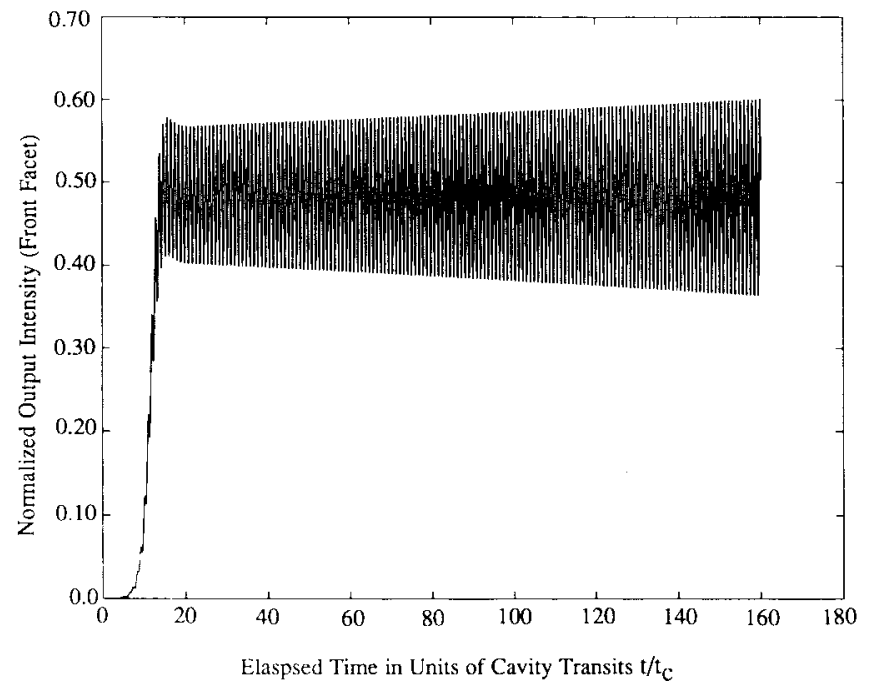

Fig. 9. Normalized output beam intensity from front facet $(|\kappa L|=2$, $\left.g_{c}=0.5,(\alpha L)_{\text {unsat }}=1.4\right)$.

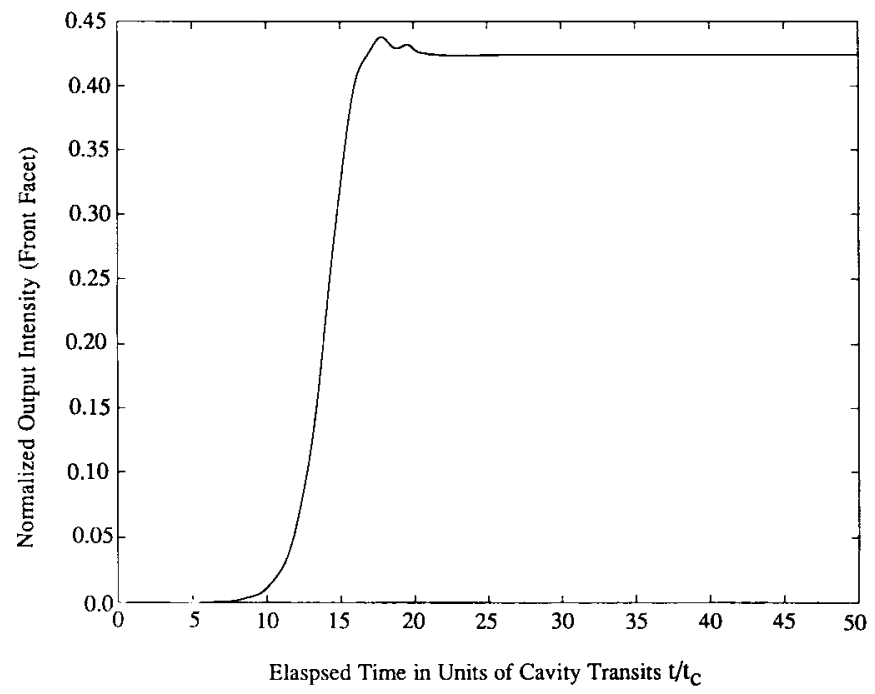

Fig. 10. Normalized output beam intensity from front facet $(|\kappa L|=2$, $\left.g_{c}=0,(\alpha L)_{\text {unsat }}=1.4\right)$.

i.e., $g_{c}=0$. Consistent with our theoretical predictions, simultaneous operation of the +1 and -1 degenerate modes is not observed.

\section{SUMMARY}

We have analyzed the multimode stability of antisymmetrically chirped index-coupled solid-state DFB lasers operating above threshold with weak to moderate gain saturation. We have demonstrated analytically that such lasers support the simultaneous lasing of their two lowest order modes. These modes are degenerate and have intensity profiles which are mirror images of one another with respect to the center of the laser cavity. Lasing of either degenerate mode by itself is unstable. The two degenerate modes beat together, producing high-frequency oscillations of the laser's output intensity. Multimode stability can be directly related to the degree by which the spatial mode profiles of the two degenerate modes overlap. A large chirp factor yields a smaller spatial overlap and a correspondingly higher degree of stability. A large chirp factor, however, also tends to reduce the modulation depth of the high-frequency oscillations. For an ideal uniform index-coupled DFB laser, neither single-mode nor dual-mode operation are stable operating regimes. A numerical simulation, based on a pair of time-dependent coupled-mode equations, quantitatively verified our analytic predictions. Finally, an exact closed-form expression for the output intensity of an antisymmetrically chirped index-coupled DFB laser, as a function of the unsaturated gain, was derived. This expression is valid for arbitrary levels of gain saturation. Work is currently underway to fabricate antisymmetrically chirped DFB lasers in rare earth-doped glass using the bent waveguide technique developed by Hillmer [26]. It follows from (2.14) and Table I that a 2-cm-long device with $g_{c}=2$ and $|\kappa L|=2$ should yield a beat frequency of approximately $20 \mathrm{GHz}$. If the nominal grating period is chosen to support lasing in the vicinity of $1.5 \mu \mathrm{m}$, then a grating chirp parameter of $g_{c}=2$ corresponds to a variation of the grating period of about $1.2 \times 10^{-2} \%$ measured from the front to the rear facet. Much higher beat frequencies, on the order of hundreds of gigahertz, should be achievable using semiconductor chirped-grating DFB lasers, which have much shorter cavity lengths [3].

\section{REFERENCES}

[1] H. Kogelnik and C. V. Shank, "Coupled mode theory of distributed feedback lasers," J. Appl. Phys., vol. 43, pp. 2327-2335, 1972.

[2] L. M. Zhang and J. E. Carroll, "Dynamics and hole burning in uniform DFB semiconductor lasers," Int. J. Optoelectron., vol. 8, pp. 279-291, 1993.

[3] Y.-H. Liao and H. G. Winful, "Extremely high-frequency self-pulsations in chirped grating feedback semiconductor lasers," Appl. Phys. Lett., vol. 69, pp. 2989-2991, 1996.

[4] D. Wake, "Optical devices for millimeter-wave transmission," in Proc. 1996 Int. Topical Meeting on Microwave Photonics (MWP'96), Kyoto, Japan, Dec. 3-5, 1996, paper WE1-1, pp. 145-148.

[5] M. Brunel, O. Emile, M. Alouini, A. L. Floch, and F. Bretenaker, "Selfmode-locked pulsed monomode laser," Opt. Lett., vol. 24, pp. 229-231, 1999.

[6] T. Fessant, "Large signal dynamics of distributed feedback lasers with spatial modulation of their coupling coefficient and grating pitch," Appl. Phys. Lett., vol. 71, pp. 2880-2882, 1997.

[7] J. O'Reilly and P. Lane, "Remote delivery of video services using mm-waves and optics," J. Lightwave Technol., vol. 12, pp. 369-375, 1994.

[8] D. Wake, C. R. Lima, and P. A. Davies, "Transmission of $60-\mathrm{GHz}$ signals over $100 \mathrm{~km}$ of optical fiber using a dual-mode semiconductor laser source," IEEE Photon. Technol. Lett., vol. 8, pp. 578-580, 1996.

[9] S. V. Chernikov, R. Kashyap, P. F. McKee, and J. R. Taylor, "Dual frequency all fiber grating laser source," Electron. Lett., vol. 29, pp. 1089-1091, 1993.

[10] E. A. Swanson and S. R. Chinn, "23-GHz and $123-\mathrm{GHz}$ soliton pulse generation using two $\mathrm{CW}$ lasers and standard single mode fiber," IEEE Photon. Technol. Lett., vol. 6, pp. 796-798, 1994.

[11] H. Kogelnik and C. V. Shank, "Stimulated emission in a periodic structure," Appl. Phys. Lett., vol. 18, pp. 152-154, 1971.

[12] C. V. Shank, J. E. Bjorkholm, and H. Kogelnik, "Tunable distributedfeedback dye laser," Appl. Phys. Lett., vol. 18, pp. 395-396, 1971.

[13] K. O. Hill and A. Watanabe, "Envelope gain saturation in distributedfeedback lasers," Appl. Opt., vol. 14, pp. 950-961, 1975.

[14] H. Haus, "Gain saturation in distributed feedback lasers," Appl. Opt. vol. 14 , pp. 2650-2652, 1975.

[15] P. Szczepanski, "Approximate analysis of nonlinear operation of a distributed-feedback laser," Appl. Opt., vol. 24, pp. 3574-3578, 1985.

[16] _ "Approximate analysis of gain saturation of a distributed feedback laser with end reflectors," IEEE J. Quantum Electron., vol. QE-22, pp. 517-519, 1986. 
[17] S. Solimeno and G. Mastrocinque, "Gain saturation and output power of distributed feedback lasers," J. Phys. A: Math Gen., vol. 9, pp. 1309-1321, 1976.

[18] M. Sargent, "Laser saturation grating phenomena," Appl. Phys., vol. 9, pp. 127-141, 1976 .

[19] M. Sargent, III, W. H. Swanter, and J. D. Thomas, "Theory of a distributed feedback laser," IEEE J. Quantum Electron., vol. QE-16, pp. 465-472, 1980 .

[20] G. A. Lyakhov and Yu. V. Ponomarev, "Steady-state nonlinear distributed feedback," Sov. J. Quantum Electron., vol. QE-6, pp. 57-59, 1976.

[21] W. S. Rabinovich and B. J. Feldman, "Spatial hole burning effects in distributed feedback lasers," IEEE J. Quantum Electron., vol. 25, pp. 20-30, 1989.

[22] W. E. Lamb, Jr., "Theory of an optical maser," Phys. Rev. A, vol. 134, pp. 1429-1450, 1964.

[23] A. E. Siegman, Lasers. Mill Valley, CA: University Science, 1986, pp. 992-1003.

[24] Y.-H. Liao, "Dynamics of distributed feedback lasers and semiconductor laser arrays," Ph.D. dissertation, Dept. of Electrical Engineering, Univ. of Michigan, Ann Arbor, 1997.

[25] M. Schubert and B. Wilhelmi, Nonlinear Optics and Quantum Electronics. New York: Wiley, 1986, ch. 1.
[26] H. Hillmer, A. Grabmaier, S. Hansmann, H.-L. Zhu, H. Burkhard, and K. Magari, "Tailored DFB laser properties by individually chirped gratings using bent waveguides," IEEE J. Select. Topics Quantum Electron., vol. 1, pp. 356-362, 1995.

Kim A. Winick (S'77-M'80-SM'98) was born in New York City, NY, on July 27, 1954. He received the B.S. degree in electrical engineering from Pennsylvania State University, State College, in 1976 and the M.S. and Ph.D. degrees in electrical engineering from the University of Michigan, Ann Arbor, in 1977 and 1981, respectively.

While at the University of Michigan, he held a National Science Foundation Graduate Fellowship. From 1981 until 1988, he was a Member of the Technical Staff at the Massachusetts Institute of Technology's Lincoln Laboratory, where he worked on millimeter-wave and coherent optical communication systems. In 1988, he joined the faculty of the Electrical Engineering and Computer Science Department at the University of Michigan. He is currently an Associate Professor. His major research interests are glass and crystal integrated optics and communication theory.

Dr. Winick is a member of the Optical Society of America. 